Cahiers $d u$ MONDE RUSSE

\section{Cahiers du monde russe}

Russie - Empire russe - Union soviétique et États indépendants

$50 / 2-3 \mid 2009$

L'Europe orientale, 1650-1730. Crises, conflits et renouveau

\title{
Archives et histoire dans les sociétés postcommunistesSonia COMBE, éd.
}

, Paris : La Découverte / BDIC, 2009, 332 p.

Duane Huguenin

\section{OpenEdition}

\section{Journals}

Édition électronique

URL : https://journals.openedition.org/monderusse/9801

DOI : 10.4000/monderusse. 9801

ISSN : $1777-5388$

Éditeur

Éditions de l'EHESS

Édition imprimée

Date de publication : 15 septembre 2009

ISBN : 978-2-7132-2260-3

ISSN : $1252-6576$

Référence électronique

Duane Huguenin, "Archives et histoire dans les sociétés postcommunistesSonia COMBE, éd. », Cahiers du monde russe [En ligne], 50/2-3 | 2009, mis en ligne le 14 janvier 2013, consulté le 03 septembre 2022. URL : http://journals.openedition.org/monderusse/9801 ; DOI : https://doi.org/ 10.4000/monderusse. 9801

Ce document a été généré automatiquement le 3 septembre 2022.

Tous droits réservés 


\title{
Archives et histoire dans les sociétés postcommunistesSonia COMBE, éd.
}

\author{
, Paris : La Découverte / BDIC, 2009, 332 p.
}

Duane Huguenin

\section{Sonia COMBE, éd., Archives et histoire dans les sociétés postcommunistes, Paris : La Découverte / BDIC, 2009, 332 p.}

Depuis la chute des régimes communistes en 1989, l'histoire du communisme a connu un profond renouvellement, du fait notamment de l'ouverture aux chercheurs des fonds d'archives auparavant secrets. Cette ouverture a souvent été très large, notamment pour les archives des partis communistes, et a d'emblée suscité un double intérêt de la part des historiens. En effet, si les archives sont pour le chercheur avant tout une source l'objet sur lequel s'appuie l'histoire qu'il écrit -, elles sont aussi en elles-mêmes un objet d'histoire, produit et révélé dans des circonstances données qui interrogent l'historien autant que le contenu des documents produits. Le culte des archives - entretenu par les régimes communistes qui les ont occultées ainsi que par de nombreux critiques du communisme qui espéraient y trouver la preuve du caractère criminel de ces régimes - a abouti après 1989 à faire disparaître, dans les débats publics tout au moins, la prise en compte des archives comme documents au profit de "révélations " fondées sur ce matériau par essence véridique qu'elles constitueraient. Le statut des archives à l'Est, dans sa matérialité et dans son apport problématique à l'écriture de l'histoire, a déjà fait l'objet de publications depuis $1989^{83}$, mais les remous de l'actualité récente (que l'on pense à l'affaire Kundera en République tchèque, qui fait l'objet de la contribution de Muriel Blaive dans cet ouvrage) et le rapide renouvellement des problématiques historiques justifiaient amplement un nouveau bilan, à un moment où la recherche historique sur le communisme semble atteindre une certaine maturité. C'est tout l'objet de ce livre, qui fait suite à un colloque tenu à l'automne 2007 à la BDIC.

2 Le livre se divise en trois parties de longueur inégale. La première, la plus courte, est consacrée à l'accès aux archives, et propose notamment un état des lieux comparatif 
indispensable à la compréhension des mécanismes par lesquels l'historien a pu accéder à ces sources nouvelles et à la perception des fortes différences nationales qui perdurent aujourd'hui dans la gestion des archives. La seconde partie, la plus importante, développe les apports historiographiques permis par l'ouverture des archives et se concentre sur quatre domaines essentiels, quoique non exhaustifs: les archives privées, celles de politique étrangère, les relations entre pouvoir et société, et la Shoah à l'Est. Enfin, la dernière partie est consacrée à l'instrumentalisation du passé après 1989 et au poids des enjeux politiques sur l'utilisation historienne des archives.

3 La première partie est composée de deux chapitres fort différents et de taille très différente. Le premier est l'œuvre de Charles Kecsméti, ancien président du Conseil international des archives, et se présente comme un état des lieux de l'ouverture des archives selon les pays depuis 1989. Si, dans tous les États, la situation s'est nettement améliorée en vingt ans, les éléments fondamentaux des recommandations du Conseil de l'Europe en matière d'accès aux archives restent inégalement respectés: l'accès aux inventaires en particulier reste difficile en Roumanie et en Bulgarie, mais surtout en Russie, et les règles de déclassification des documents secrets sont, dans ces mêmes pays, bien plus incertaines qu'en Allemagne, Slovaquie et Hongrie. L'Allemagne jouit, d'une manière générale, d'une situation nettement privilégiée par rapport à ses voisins de l'Est.

Le second chapitre, l'un des plus intéressants de l'ouvrage, est l'œuvre de Victoria Prozorova-Thomas et consacré à l'ouverture contrariée des archives soviétiques à Moscou. L'auteur examine le problème du côté des archivistes et des différentes lois sur les archives. Elle met à jour la complexité des enjeux professionnels et politiques concernant la gestion des archives secrètes et montre comment les choix retenus (déclassification document par document notamment) pénalisent le processus d'ouverture et favorise l'obstruction quasi systématique mise en œuvre par les grands ministères (Justice, Défense, Intérieur), ceux-ci réussissant largement à conserver leurs propres fonds, avec des règles d'accès $a d$ hoc qui contribuent à en limiter l'accès.

La deuxième partie est consacrée aux apports historiographiques liés à l'ouverture des archives et, dans un premier temps, des archives privées, avec l'étude de Bernard $\mathrm{H}$. Bayerlein sur les journaux de Georgij Dimitrov et d'Ivan Majskij. Ces deux journaux sont très différents, à la fois dans leur parcours en tant que document, dans leur contenu et dans leur forme. Pourtant, ils peuvent s'analyser en parallèle comme deux types d'attitudes vis-à-vis du stalinisme et comme deux regards sur la politique extérieure soviétique - le premier journal étant centré sur l'action clandestine du Komintern dirigée par un homme en contact permanent avec Stalin, le second sur le travail officiel d'un ambassadeur en Grande-Bretagne impliqué dans les négociations de "fronts antifascistes ». Ces journaux permettent ainsi de remettre efficacement en perspective les archives dans leur double rôle de source et d'objet d'étude.

Dans cette même ligne, le chapitre de Jean-François Fayet consacré aux fonds personnels dans les centres d'archives soviétiques représente un exemple intéressant de retournement de l'intérêt de l'historien. En effet, déçu de ne pas trouver dans les fonds personnels qu'il souhaitait étudier le matériau qu'il espérait, l'auteur a réorienté son étude vers l'analyse de la constitution même, fort complexe, de ces fonds personnels (rares), dont l'existence est souvent étroitement liée à la répression (ou à la crainte de la répression) dont les titulaires ont été victimes. Ici, la source espérée se révèle plus riche pour ce qu'elle est que pour ce qu'elle dit, et permet d'éclairer le fonctionnement de la bureaucratie communiste sous un angle très intéressant. 
$7 \mathrm{Au}$ regard de ces deux chapitres sur les archives privées, ceux consacrés à la politique étrangère des démocraties populaires - rédigés par Antoine Marès (Tchécoslovaquie), Irina Gridan (Roumanie), Pierre Jardin (RDA) et Maria Pasztor (Pologne) - laissent le lecteur sur sa faim. La frustration tient d'abord à l'angle choisi par ces chapitres : tout à leur description des archives permettant de faire une histoire renouvelée de la politique extérieure des pays communistes, les auteurs ne développent que très ponctuellement les avancées réelles accomplies dans ce domaine et se contentent fréquemment de lister les sources et leurs enjeux sans les confronter directement à l'analyse historique qu'on pourrait en tirer. Par ailleurs, chaque chapitre bute inévitablement sur l'aporie des archives soviétiques, quasiment inaccessibles au public et qui empêchent au final toute recherche globale et aboutie sur le degré de dépendance de chaque démocratie populaire vis-à-vis de Moscou.

8 Troisième domaine de renouvellement historiographique (au sein de la deuxième partie), les relations entre l'État-parti et la société font l'objet de cinq chapitres très hétérogènes. L'introduction de Paul Gradwohl souligne avec une grande finesse les enjeux de l'ouverture des archives pour repenser les relations entre pouvoir et société sous le communisme. L'auteur souligne d'abord que le premier réflexe des chercheurs chercher la vérité dans les archives - s'est heurté à un mur évident : il n'y a pas de source qui parle d'elle-même, sans questionnement préalable apporté par l'historien; car c'est bien, au-delà de l'ouverture des archives, le renouvellement des questionnements qui a permis à l'histoire du pouvoir communiste d'être appréhendée avec des yeux nouveaux. Ces nouvelles problématiques s'articulent autour des modalités du contrôle social, mais aussi de ses limites ou de ses apories. Le communisme s'est en effet caractérisé par une culture politique spécifique où le pouvoir tente de limiter au maximum les incertitudes par un contrôle social et économique pesant, mais dont l'efficacité n'est pas garantie. Dariusz Jarosz montre ainsi l'incapacité du pouvoir polonais à répondre au " problème de la viande » malgré la multiplicité des solutions mises en œuvre (allant de la répression à la publication de livres de recette végétariens). Problème social à part entière, la pénurie de viande devient un lieu de cristallisation du mécontentement social et de l'action politique, rappelant que la rencontre entre le pouvoir et la population se fait parfois dans des lieux bien moins encadrés qu'un commissariat ou qu'une cellule du parti, comme les queues devant les magasins.

Par ailleurs, le contrôle social sécrète au sein des sociétés communistes différenciation et fragmentation. Andrea Petö, revenant dans sa contribution sur les discussions menées lors du colloque, souligne avec beaucoup de justesse que les mécanismes de création et de structuration des divers groupes sociaux par un pouvoir prônant une société sans classe constituent un enjeu essentiel et encore inégalement pris en compte de l'histoire sociale du communisme; et qu'à ce titre, l'histoire du genre, notamment, serait une clé de lecture essentielle de cette dernière. L'article de Sergueï Krasilnikov sur les groupes marginaux déportés dans l'URSS stalinienne montre également comment la répression et la mortalité bouleversent la composition traditionnelle des populations déportées, avant que les mécanismes d'adaptation ne soient mis en œuvre et ne permettent un retour à la normale, mais dans une réalité sociale profondément transformée.

Cette partie s'achève par un chapitre de Muriel Blaive consacré à « l'affaire Kundera » en Tchécoslovaquie. À partir des mécanismes qui ont déclenché le scandale (révélations issues d'archives inédites indiquant que Kundera aurait dénoncé un homme à la police tchécoslovaque), l'auteur revient sur l'usage public des archives de police en République 
tchèque et sur la manière dont la culture du sensationnalisme et de la dénonciation a largement étouffé le recul nécessaire à l'exercice consciencieux du métier d'historien.

Dernier domaine de renouvellement historiographique, la Shoah à l'Est a fait l'objet d'un ouvrage récent ${ }^{84}$ et d'un colloque à Kiev ${ }^{85}$, qui ont permis de montrer l'apport de sources nouvelles - en particulier orales - avec l'enquête du père Dubois auprès d'Ukrainiens ayant assisté ou participé à la Shoah par balles. Antonella Salomoni, notamment, montre que les archives soviétiques permettent entre autres de déconstruire le mythe de la passivité juive face au nazisme et de montrer, malgré les tabous officiels, la participation importante des juifs à la résistance contre les Allemands.

Enfin, la dernière partie de l'ouvrage est consacrée aux enjeux mémoriels et politiques liés aux archives du communisme. Les différents chapitres soulignent l'importance de ces enjeux, qui expliquent bien souvent l'intérêt du monde politique pour ces archives et en rendent l'exploitation difficile (pour faire obstruction à la révélation de la vérité sur le passé des nouveaux dirigeants bulgares, affirme par exemple Mona Foscolo), tant d'un point de vue matériel (accès réglementé) que conceptuel (prégnance des attentes sociales). Ces contributions aboutissent toutes à la conclusion qu'au-delà des retombées politiques, notamment en termes de "lustration ${ }^{86}$, l'un des problèmes persistants réside dans les conditions matérielles d'accès aux archives qui, faute de locaux, d'argent, de temps, ne permettent qu'une avancée lente de la recherche, pourtant nécessaire pour dépassionner certains débats et permettre aux sociétés postcommunistes de regarder leur passé avec objectivité et sérénité.

13 Au-delà de l'inévitable hétérogénéité des contributions qui est le propre de tous les ouvrages collectifs, on soulignera deux regrets que cet ouvrage suscite. D'une part, la confrontation entre archivistes et historiens est inégalement aboutie et aurait mérité plus de place pour resituer l'enjeu des archives non seulement dans un cadre politique, mémoriel et historien, mais également matériel en termes de gestion de la masse des documents rendus brutalement consultables. D'autre part, on aurait souhaité que le travail comparatiste soit plus développé ; et qu'à l'image du travail de Muriel Blaive sur la Tchécoslovaquie ou des propos de Thomas Lindenberger sur la RDA, des liens clairs soient établis entre les différentes configurations évoquées pour comprendre comment, dans chaque pays, se structurent des climats politiques et des attentes sociales qui pèsent inévitablement sur le débat historique et sur l'émergence d'historiographies qui, certes, échangent entre elles mais restent encore trop souvent cloisonnées. La frontière entre pays postcommunistes et anciennes républiques soviétiques pourrait à ce titre faire l'objet d'une analyse critique plus poussée.

\section{NOTES}

83. Genèses, « Les archives de l'Est », 52, 2003.

84. Antonella Salomoni, L'Union soviétique et la Shoah, P. : La Découverte, 2008. 
85. Colloque « La Shoah par balles en Ukraine » organisé par la Sorbonne, le Mémorial de la Shoah et le United Holocaust Memorial de Washington les 1-2 octobre 2007.

86. Des lois de lustration ont été prises dans divers pays postcommunistes : la lustration consiste à vérifier si certaines personnes (fonctionnaires, ou hommes politiques, selon les pays) ont par le passé servi d'informateur à la police secrète. 